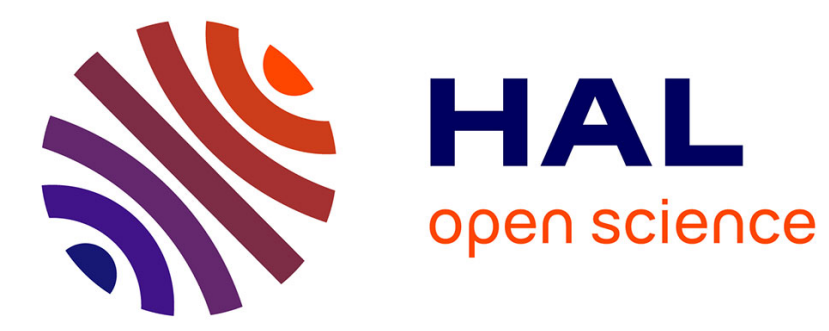

\title{
Diffuso-mechanical coupling in cholesteric liquid crystals
} I. Jánossy

\section{To cite this version:}

I. Jánossy. Diffuso-mechanical coupling in cholesteric liquid crystals. Journal de Physique Lettres, 1981, 42 (2), pp.41-43. 10.1051/jphyslet:0198100420204100 . jpa-00231869

\section{HAL Id: jpa-00231869 https://hal.science/jpa-00231869}

Submitted on 1 Jan 1981

HAL is a multi-disciplinary open access archive for the deposit and dissemination of scientific research documents, whether they are published or not. The documents may come from teaching and research institutions in France or abroad, or from public or private research centers.
L'archive ouverte pluridisciplinaire HAL, est destinée au dépôt et à la diffusion de documents scientifiques de niveau recherche, publiés ou non, émanant des établissements d'enseignement et de recherche français ou étrangers, des laboratoires publics ou privés. 


\title{
Diffuso-mechanical coupling in cholesteric liquid crystals
}

\author{
I. Jánossy \\ Central Research Institute for Physics, H-1525 Budapest, P.O.B. 49, Hungary \\ (Reçu le 13 octobre 1980, accepté le 27 novembre 1980)
}

\begin{abstract}
Résumé. - L'étude d'une suspension de petites particules dans un cholestérique en écoulement de cisaillement met en évidence une dérive transversale de ces petites particules. Des mesures de la valeur de la vitesse de dérive sont présentées. Ce phénomène est interprété en utilisant l'analogie du couplage thermomécanique prévu pour les cholestériques.
\end{abstract}

\begin{abstract}
It has been observed that in cholesterics shear flow induces a transverse drift of small particles suspended in the liquid crystal. Quantitative measurements of the drift velocity are presented. The effect is interpreted using the analogy of the thermomechanical coupling, predicted for cholesterics.
\end{abstract}

1. Introduction. - In a recent publication [1] we called attention to some original flow properties of cholesteric liquid crystals, which are closely connected with the lack of mirror symmetry in these systems. One of these effects, the occurrence of a shear flow induced instability involving propagating domains, has been discussed in detail in [2]. In this letter we consider the second effect mentioned in reference [1], that is the shear flow induced transverse drift of small particles suspended in a cholesteric.

As described in reference [1], the effect was observed in a torsional shear apparatus [3]. The cholesteric was placed between two parallel disks; the shear flow was produced by rotating one of the disks, keeping the other one fixed. It was found that for one sense of rotation the particles present in the sample drifted inward and accumulated at the centre of the disks. On reversing the sense of rotation the particles were swept out from the centre. This observation indicates that in cholesterics the flow induces a diffusion of particles, relative to the liquid crystal, the direction of diffusion being perpendicular to the flow and to the velocity gradient.

The aim of the present letter is to give a more quantitative description of this induced diffusion.

2. Determination of the drift velocity. - In order to carry out quantitative measurements, we introduced into the cholesteric small glass spheres with diameters between 5 and $10 \mu \mathrm{m}$. The concentration of particles was of the order of $10^{5}$ particles $/ \mu \mathrm{m}^{3}$. The measurements consisted of determining the flow induced change of concentration of the particles at certain points in the sample. The concentration was determined by counting the number of particles within a given area.

We shall now show how the drift velocity can be calculated from the concentration data. The sample can be regarded as a two-component system : liquid crystal + particles. The continuity equation holds separately for the two components; for the particles it can be written as

$$
\frac{\partial c}{\partial t}=\operatorname{div} c v_{\mathrm{t}}=\frac{1}{r} \frac{\partial}{\partial r} r c v_{\mathrm{t}}
$$

where $r$ is the distance from the centre, $c$ and $v_{\mathrm{t}}$ are the concentration and the radial drift velocity respectively, averaged over the distance from the walls of the sample and the azimuthal angle. (We take $v_{\mathrm{t}}$ to be positive for particles drifting inward.)

Let us suppose that $v_{\mathrm{t}}$ is proportional to the shear rate $s$, also averaged over the distance from the disks :

$$
v_{\mathrm{t}}=\lambda s
$$

where $\lambda$ is a coupling coefficient with the dimensions of length. In terms of the sample thickness $L$ and the angular velocity $\Omega$ of the rotating disk (taken to be positive when the disk, viewed through the liquid crystal, rotates counter-clockwise), we have $s=\Omega r / L$ so that equation (1) can be written as :

$$
\frac{\partial c}{\partial t}=\frac{\Omega}{L} \lambda\left(2 c+r \frac{\partial c}{\partial r}\right)
$$


For infinite disks with a uniform distribution of particles at $t=0$, the solution of equation (3) is :

$$
c(r, t)=c(t)=c_{0} \mathrm{e}^{t / \tau}
$$

with $\tau=L / 2 \lambda \Omega$.

According to equation (4) the flow changes the concentration of particles, but does not create any concentration gradients in the sample. This is valid only if :

a) the linear relation between $s$ and $v_{t}$ holds,

b) the influence of the finite size of the disks can be neglected.

For $\tau<0$, the particles move outward and the finite size has an effect only near the edge of the disks. On the other hand, for $\tau>0$ the particles move inward and we cannot expect equation (4) to hold at any given $r$ after such time as is necessary for a particle to drift from the boundary to that radius. For large $t$ the particles will be concentrated at the centre; this was the effect originally observed [1]. For quantitative measurements it is however more convenient to investigate the concentration variation for $\tau<0$, or in the case of $\tau>0$ only for small $t$, and to evaluate $\lambda$ with the help of equation (4).

3. Experimental results. - The disks were in most experiments rubbed tangentially to provide a planar texture. However as the flow always created many disclinations the measurements were carried out in a strongly perturbed configuration.

In figures 1 and 2 measurements on MBBA $+1 \%$ cholesteryl nonanoate are presented (pitch $P=-12 \mu \mathrm{m}, L=50 \mu \mathrm{m}$ ). The flow caused a change in the concentration but did not create a significant concentration gradient in the sample. This fact indicates that the linear relation between $s$ and $v_{\mathrm{t}}$ is more or less satisfied, at least up to $s \approx 10 \mathrm{~s}^{-1}$. From the time dependence of the concentration we get, with the help of equation (4), $\lambda=-135 \mathrm{~nm}$.

We note that the value of $\lambda$ was not very reproduci-

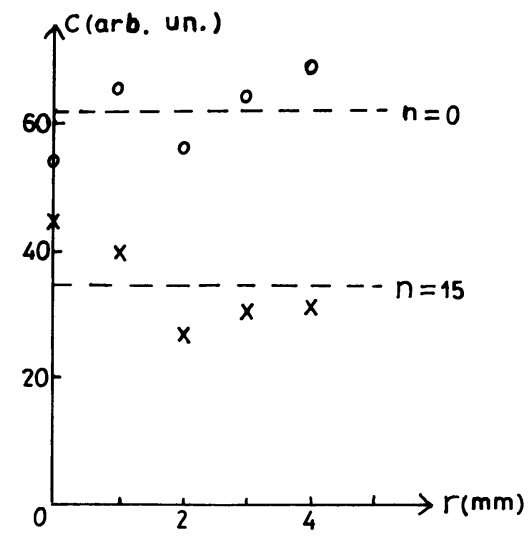

Fig. 1. - The concentration of particles as a function of the distance from the centre at $t=0$, and after 15 turns of the disk. Time of revolution $50 \mathrm{~s}$. The average shear rate at $r=4 \mathrm{~mm}$ was $10 \mathrm{~s}^{-1}$; the radius of the disks was $12 \mathrm{~mm}$.

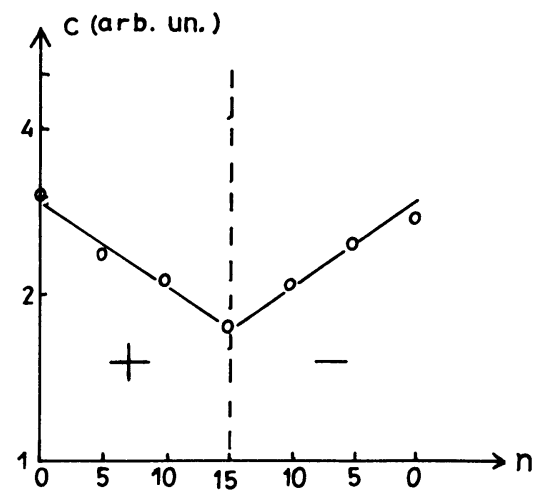

Fig. 2. - Variation of the concentration as a function of the number of turns. The turns are counted to be positive when the disk, viewed through the liquid crystal, rotates counterclock-wise.

ble; the values obtained from different measurements deviated by as much as $20-30 \%$. In spite of this uncertainty the following features emerge quite clearly :

1) There is no effect in the isotropic or nematic phase. This fact is illustrated for the isotropic phase in figure 3. From similar measurements we obtained $|\lambda|<5 \mathrm{~nm}$ for pure MBBA.

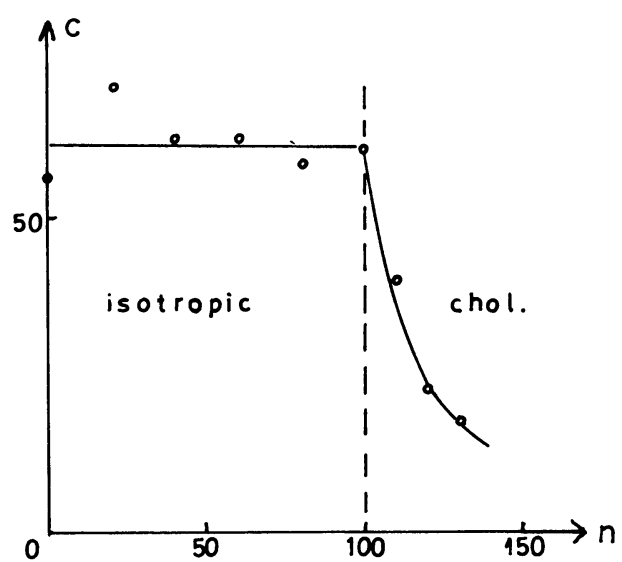

Fig. 3. - Variation of the concentration as a function of the number of turns. For $n<100$ the sample has been kept in isotropic phase, for $n>100$ it has been in cholesteric phase.

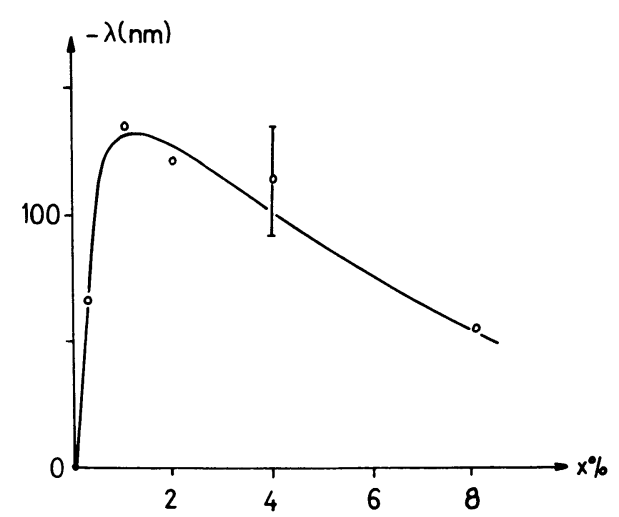

Fig. 4. $-\lambda$ as a function of the weight percent of cholesteryl nonanoate $(x)$ in MBBA. 
2) On reversing the sign of the pitch, $\lambda$ changes sign too. For example we found, for MBBA doped with $5 \%$ CB 15 (BDH product), that $\lambda=+66 \mathrm{~nm}$ $(P \approx+3 \mu \mathrm{m})$.

3) Varying the pitch of the liquid crystal by doping a nematic with different amounts of chiral additive, $\lambda$ exhibits a maximum at a certain pitch. This is shown in figure 4.

The maximum corresponds to $P \approx 10 \mu \mathrm{m}$, thus the effect is strongest when the pitch and the particle size are comparable.

4. Discussion. - In reference [1] we interpreted the effect described above by the analogy of the thermomechanical effect for cholesterics [4]. We have shown for the case of an undistorted helix, that the thermomechanical coupling leads to a transverse heat flux which is proportional to the shear rate. This is the analogy of equation (2), which we used here to interprete the observations. On the basis of this analogy we propose the name diffuso-mechanical coupling for the phenomenon.

This coupling cannot exist in the nematic phase because there is a centre of inversion. It is also excluded for isotropic liquids (chiral or non chiral) since in isotropic systems irreversible processes with different tensorial characters cannot couple [5]. The experimental findings presented above are in agreement with these predictions.

For particles which are much larger than molecular dimensions it should be possible to describe the diffusomechanical coupling in terms of the continuum theory of cholesterics, i.e. to calculate $\lambda$ as a function of the elastic constants, the viscosity coefficients and the pitch [7]. Without any detailed calculation the following can be established :

a) If the sign of the pitch is reversed, while the other constants remain practically unchanged, $\lambda$ changes its sign. This was indeed observed (see $\S 3$ ).

b) When the pitch is much larger than the particle size, the fluid surrounding the particle can be regarded as practically a nematic phase, so the coupling must be weak. On the other hand, when the pitch is much smaller than the particle, the cholesteric which surrounds the particle can be regarded from the point of view of hydrodynamical properties as a smectic $\mathrm{A}$ phase [6]. In this limit the coupling again becomes weak, because in a smectic A phase there cannot be any coupling between shear flow and vectorial transport processes [6]. Consequently the effect should be strongest when the particle size and the pitch are comparable, as found above (Fig. 4).

Finally we note that the diffuso-mechanical effect should also occur for the molecules themselves i.e. the flow should induce a transverse diffusion of the chiral molecules relative to the nematic matrix. We note also that the coupling coefficient for a chiral molecule and its mirror image should have the opposite sign. Thus dissolving a racemic mixture of chiral molecules in a liquid crystal, the flow induced diffusion will be of opposite direction for the two optical isomers; i.e. the flow will tend to separate them. The corresponding coupling coefficient however may be several orders of magnitude smaller than the one observed for macroscopic particles.

\section{References}

[1] JÁNossy, I. in Proceedings of the Third Liquid Crystal Conf. of Socialist Countries (1979).

[2] JÁnossy, I., J. Physique 41 (1980) 437.

[3] WAHL, J. and FiSCHER, F., Mol. Cryst. Liq. Cryst. 22 (1973) 359.

[4] Leslie, F. M., Proc. R. Soc. A 307 (1968) 359 and Symp. Faraday Soc. 5 (1971) 33 ;

Chandrasekhar, S., Liquid Crystals (Cambridge) 1977.

[5] De Groot, S. and MazUR, P., Non-Equilibrium Thermodynamics (North-Holland, Amsterdam) 1962.
[6] Martin, P. C., Parodi, O. and Pershan, P. S., Phys. Rev. A6 (1972) 2401.

[7] An attempt to explain the coupling in terms of continuum theory has recently been given by CHANDRASEKHAR, S. Kini, U. D., and Ranganath, G. S., Proceedings of the International Liquid Crystal Conference, Bangalore, (1979) p. $247-253$. 